University of Massachusetts Amherst

ScholarWorks@UMass Amherst

Chemistry Department Faculty Publication Series

Chemistry

1993

\title{
Flow Injection Systems for Directly Coupling On- line Digestions with Analytical Atomic Spectrometry Part 2. Reactions in a Microwave Field
}

Julian Tyson

University of Massachusetts Amherst

Thomas J. Gluodenis Jr.

University of Massachusetts Amherst

Follow this and additional works at: https://scholarworks.umass.edu/chem_faculty_pubs

Part of the Chemistry Commons

\section{Recommended Citation}

Tyson, Julian and Gluodenis, Thomas J. Jr., "Flow Injection Systems for Directly Coupling On-line Digestions with Analytical Atomic Spectrometry Part 2. Reactions in a Microwave Field" (1993). Journal of Analytical Atomic Spectrometry. 1309.

Retrieved from https://scholarworks.umass.edu/chem_faculty_pubs/1309 


\title{
Flow Injection Systems for Directly Coupling On-line Digestions With Analytical Atomic Spectrometry Part 2*. Reactions in a Microwave Field $\dagger$
}

\author{
Thomas J. Gluodenis, Jr., and Julian F. Tyson‡ \\ Department of Chemistry, University of Massachusetts, Amherst, MA 01003, USA
}

\begin{abstract}
A flow injection system incorporating a stopped-flow microwave-heated reactor was constructed for the preparation of solutions for subsequent analysis by atomic spectrometric techniques. Slurry samples were injected into the manifold and transported into a glass reactor mounted inside a microwave oven. Nitric acid was flushed into the reactor, which was then sealed and the contents were heated by an appropriate microwave programme so that the pressure was held at $400 \mathrm{lb}$ in ${ }^{-2}$ for $5 \mathrm{~min}$. During this period the presssure was continuously monitored. After cooling, the reactor was vented and the contents were flushed out into a calibrated flask and diluted to volume. The procedure was evaluated by the determination of some minor elements in cocoa powder (for which the results were compared with those of two other digestion procedures), Horse Kidney (International Atomic Energy Agency Certified Reference Material $\mathrm{H}-8$ ) and coal [National Institute of Standards and Technology Standard Reference Material 1632b Trace Elements in Coal (Bituminous)]. A comparison of the results showed no significant difference $(95 \%$ confidence) for the trace element contents of these materials between the flow injection method and other two methods of sample preparation. An analysis of variance showed no indication of sample slurry inhomogeneity. Low recoveries were obtained for the coal material, perhaps owing to incomplete dissolution of the silicate constituents.
\end{abstract}

Keywords: Flow injection; slurry sampling; on-line pressure digestion; microwave digestion; stopped flow

The introduction of samples into various types of atomic spectrometers for elemental determinations is still a topic of considerable research interest. ${ }^{1}$ Much of this interest is centred around the problems posed by solid materials. The direct introduction of solids, although overcoming the well documented disadvantages of the alternative route of dissolution, is still largely at the stage described by Greenfield et al. ${ }^{2}$ as 'singularly unsuccessful' because of problems associated with 'heterogeneity of composition, density and particle size together with impaction and selective volatilization, depending on whether the sample is powdered or a solid lump.' Hence, most analytical methods contain procedures for sample dissolution. Many such procedures require aggressive reaction conditions to achieve dissolution on a reasonable time scale. Even so, procedures are often tedious and time consuming and in need of constant supervision by laboratory staff. The automation of these dissolution procedures is clearly desirable. Flow-based techniques have proved to be an extremely versatile basis for the adaptation of existing manual procedures to an automated format ${ }^{3}$ and for the development of new analytical procedures. ${ }^{4}$ Flow techniques have been used for a number of sample pretreatment procedures coupled with atomic spectrometric determinations, ${ }^{5}$ and a number of publications recently have described approaches to the development of flowbased digestion, leaching and dissolution.

In Part $1,^{6}$ the performance of a simple flow injection (FI) system for the pressurized dissolution of cocoa powder was described. In this previous system the thermal energy was supplied by resistive heating elements and the reactor consisted of a coil of poly(tetrafluoroethylene) PTFE tubing. Disadvantages were that $(a)$ as the flux of thermal energy was continuous, the residence time within the heated region was limited by the need to vent the reactor before catastrophic failure of the tubing occurred owing to

\footnotetext{
*For Part 1 of this series, see ref. 6.

†Presented at the Sixth Biennial National Atomic Spectroscopy Symposium, University of Plymouth, UK, July 22-24, 1992.

$¥$ To whom correspondence should be addressed.
}

the pressure rise, $(b)$ as there was no 'headspace' within the reactor, the pressure rise was rapid compared with the time required for complete digestion and $(c)$ there was no measurement of the pressure within the system owing to the lack of a suitable transducer.

As a first stage in the development of a system with control over residence time, the use of microwave heating was investigated, the salient feature of such an approach being that the energy flux into the sample is under the control of the operator.

Several workers have reported on the use of on-line microwave heating as part of a sample preparation procedure. Some of these workers have been concerned with the acceleration of a homogeneous reaction by the judicious application of some heat, ${ }^{7-10}$ the determination of total phosphorus in waters, ${ }^{7}$ determination of chemical oxygen demand $(\mathrm{COD})^{8}$ and the determination of various mercury compounds in waters. ${ }^{9,10}$

A limited number of papers have reported the use of microwave heating for acid attack on some solid sample materials, a number of which described reactions at atmospheric pressure and others the use of elevated pressures $^{11-15}$ for the analysis of sewage sludge ${ }^{13}$ and some botanical and biological materials. ${ }^{14,15}$

In this paper further developments of a high-pressure, stopped-flow system for directly coupling sample dissolution with an atomic spectrometer are reported. The aims of the work were to develop procedures that are rapid and that produce total dissolution with a minimum amount of residual dissolved carbon (for samples that contain unwanted organic material). It was considered appropriate to maintain the use of pressures up to $450 \mathrm{lb}^{-2}$, but as a consequence it was also considered necessary that some measure of the pressure in the system was made as this is clearly a parameter of considerable practical importance. The system is based on the use of a small reactor vessel located in a laboratory microwave oven into which slurry samples are introduced by an FI sample and reagent handling system. The procedure was evaluated for the dissolution of some well characterized materials with a considerable amount of organic matrix. 
Table 1 Instrumental parameters for the determination of trace elements in cocoa powder and Horse Kidney by flame AAS

\begin{tabular}{lccccc}
\multicolumn{1}{c}{ Parameter } & Fe & $\mathrm{Zn}$ & $\mathrm{Cd}$ & $\mathrm{Mg}$ & $\mathrm{Ca}$ \\
Wavelength/nm & 248.3 & 213.9 & 228.8 & 202.6 & 422.7 \\
Lamp current/mA & 30 & 15 & 8 & 10 & 10 \\
Spectral bandpass/nm & 0.2 & 0.7 & 0.7 & 0.7 & 0.7 \\
Acetylene flow rate/l min m $^{-1}$ & 2.0 & 2.0 & 2.0 & 2.0 & 2.0 \\
Air flow rate/l min & 8.0 & 8.0 & 8.0 & 8.0 & 8.0 \\
Burner height (arbitrary units) & 8 & 8 & 8 & 8 & 8 \\
No. of replicates & 3 & 3 & 3 & 3 & 3 \\
Integration time/s & 3 & 3 & 3 & 3 & 3
\end{tabular}

Table 2 Instrumental parameters for the determination of iron in cocoa powder by ICP-AES

$\begin{array}{lc}\quad \text { Parameter } & \text { Value } \\ \text { R.f. frequency } / \mathrm{MHz} & 27.12 \\ \text { Incident power } / \mathrm{kW} & 1.0 \\ \text { Argon gas flow rates } / 1 \mathrm{~min}^{-1} & \\ \text { Outer } & 15 \\ \text { Intermediate } & 1.0 \\ \text { Nebulizer } & 0.7 \\ \text { Observation height/mm } & 15 \\ \text { Read delay/s } & 20 \\ \text { Sampling time } / \mathrm{ms} & 100 \\ \text { Analytical line/nm } & 238.204 \\ \text { Reference line } / \mathrm{nm} & 424.683\end{array}$

\section{Experimental}

\section{Apparatus}

\section{Spectrometers}

A Perkin-Elmer 1100B flame atomic absorption spectrometer equipped with Perkin-Elmer data-coded hollow cathode lamps was used with deuterium-arc background correction for all determinations. Instrumental parameters are given in Table 1. The signals were recorded on an Epson LQ-850 printer, all measurements being expressed as peak height absorbance.

A Plasma II ICP-AES (Perkin-Elmer) operated using Myers-Tracy signal compensation was used for the determination of iron in cocoa powder. Instrumental parameters are given in Table 2 .

\section{Pressure transducer}

The pressure transducer, designed and constructed in-house (see Fig. 1), consisted of separate PTFE and stainless-steel components and a $500 \mathrm{lb} \mathrm{in}^{-2}$ pressure gauge (Cole Parmer Model L-68800-46). The face of the transducer was a

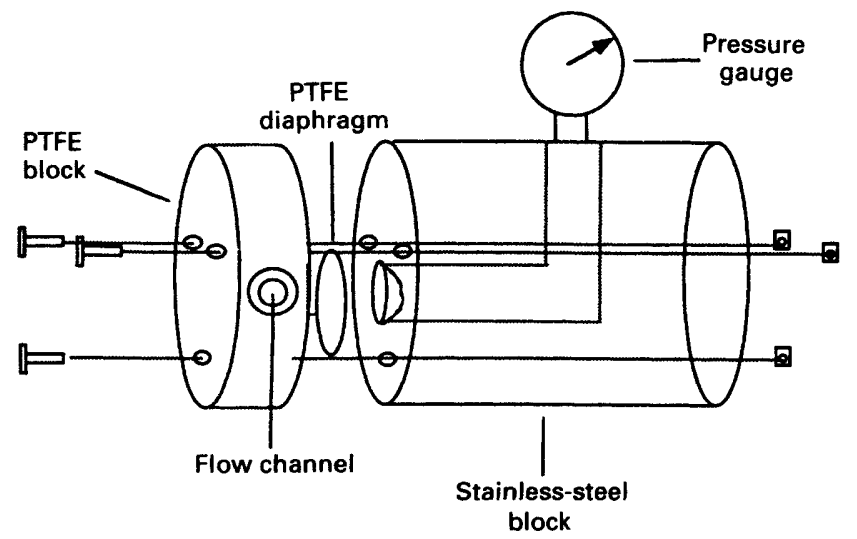

Fig. 1 Diagram of the flow-through pressure transducer circular PTFE block, 0.450 in wide, having a diameter of 1.50 in. A centre channel of diameter 0.60 in and 0.030 in deep was machined through the centre of the block. Two $1.5 \mathrm{~mm}$ i.d. flow channels were drilled $180^{\circ}$ through the centre to the edge of the block and terminated in 1/4-28 male end fittings compatible with Omnifit Model 2210 fittings. Four bolt holes, of diameter 0.174 in, were drilled through the width of the block for assembly of the transducer. A stainless-steel face-plate, 0.100 in thick was fabricated to fit over the face of the block to prevent distortions of the PTFE as the pressure transducer was assembled and tightened down.

The body of the transducer was a 2.0 in length of stainless-steel round stock, of diameter $2.75 \mathrm{in}$. The front face of the block contains a 0.60 in diameter hemi-spherical depression 0.5 in deep to allow for membrane distortion. A 0.004 in deep recess having a diameter of 1.320 in is also machined into the front of the transducer so as to accommodate a PTFE membrane of diameter 0.710 in and thickness 0.005 in. A 0.25 in diameter channel extends 0.5 in from the bottom of the depression on the face of the block towards the centre of the block to form a right-angle with a second channel of the same diameter extending outward from the centre of the block to the edge. This second channel is threaded with a 0.25 in non-parallel thread to accommodate the pressure gauge. Four bolt holes, passing through each face of the transducer body, extend the length of the stainless-steel block. They are of diameter 0.165 in to a depth of 1.750 in, and then drilled to a diameter of 0.136 in for the remaining depth of 0.025 in, which was threaded for 8-32 threads in ${ }^{-1}$. The bolts consist of 3.0 in long stainless-steel threaded rods, 8-32 threads in $^{-1}$, with an 8-32 hexagonal nut welded on one end. Calibration of the pressure transducer was performed against a $1000 \mathrm{lb}^{-2}$ pressure gauge, which was pressurized simultaneously with the developed transducer using an HPLC pump.

\section{Flow manifold}

Microwave digestions were carried out using the CEM MDS-81 microwave oven described previously. ${ }^{6}$ The manifold used is shown in Fig. 2. An Ismatec MS-4 Reglo/8-100 variable-speed, multichannel peristaltic pump was used with Viton pump tubing (Cole-Parmer) and Techicon AutoAnalyzer pump tubing. Slurry introduction was implemented through two four-way Tefzel slider valves with internal flow channels drilled to $1.5 \mathrm{~mm}$ i.d. The total volume of the valve and injection loop was $204 \mu \mathrm{l}$. The oven contained a $10 \times 1 \mathrm{~cm}$ i.d. borosilicate glass column equipped with PTFE end fittings (Omnifit Model 446121). The valves used to contain the digesting sample were Omnifit Model 1103 three-way valves mounted on either end of the column and upstream of the slider valve, and an Omnifit two-way Model 1101 valve mounted upstream of the flow-through pressure transducer. The remaining manifold tubing consisted of $0.8 \mathrm{~mm}$ i.d. PTFE tubing. All 


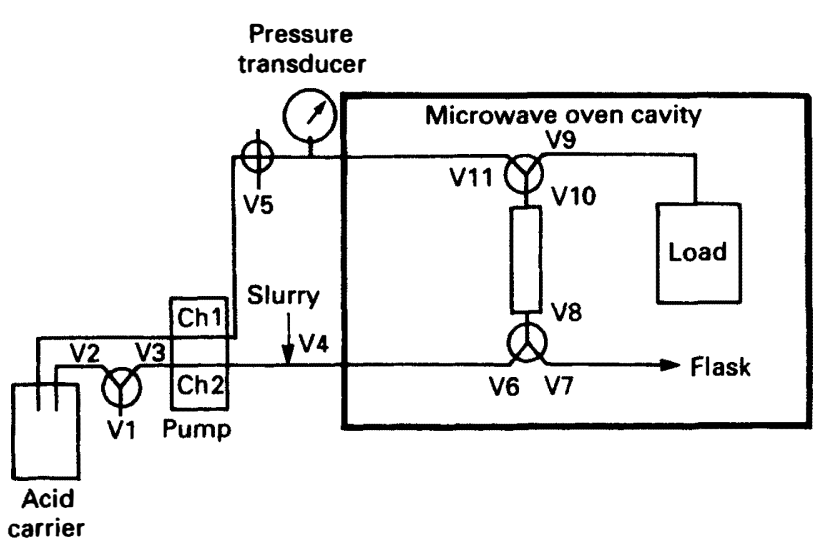

Fig. 2 Manifold for the stopped-flow microwave digestion of slurry samples (see text for details of operation)

manifold channels were connected using high-pressure gripper fittings (Omnifit).

\section{Reagents}

Analytical-reagent grade water produced by a Barnstead Epure system was used for the preparation of all solutions and as a carrier stream to the flame atomic absorption spectrometer unless stated otherwise. Copper, iron, magnesium, calcium and zinc working standard solutions were prepared by dilution of stock standard solutions (Fisher) containing $1000 \mathrm{mg} \mathrm{1}^{-1}$ of the appropriate element. Cadmium working standard solutions were prepared by dilution of $1025 \mathrm{mg} \mathrm{l}^{-1}$ cadmium stock standard solution (Aldridge). Digestions were carried out using nitric acid (Fisher, reagent grade) and sulfuric acid (Fisher, reagent grade). Lanthanum solutions $(5 \% \mathrm{~m} / \mathrm{v})$ were prepared by dissolution of $\mathrm{LaCl}_{3} \cdot 7 \mathrm{H}_{2} \mathrm{O}$ (Fisher atomic absorption grade) in analytical-reagent grade water. Triton X-100 solutions $(0.04 \%)$ were prepared by dilution of Triton X-100 (Rohm and Hass, registered trademark for octylphenoxylpolyethoxyethanol, Banco standardized, Anderson Laboratories) with $5 \% \mathrm{HNO}_{3}$. The sample materials were Hershey's cocoa powder batch number 76P, International Atomic Energy Agency (IAEA) certified reference material Horse Kidney H-8 and National Institute of Standards and Technology (NIST) Standard Reference Materials (SRMs) SRM 1632b Trace Elements in Coal (Bituminous), SRM 1575 Pine Needles, SRM 1577a Bovine Liver and SRM 1577a Oyster Tissue.

\section{Procedures}

\section{Hot-plate digestion}

The method used for the open-vessel hot-plate digestion of cocoa powder was that described previously. 6 The resulting digests were diluted to volume in $100 \mathrm{ml}$ calibrated flasks and analysed by flame AAS against acid-matched standards.

\section{Dry ashing}

Three different protocols for the dry ashing of organic samples were evaluated ${ }^{16-18}$ for the decomposition of cocoa powder. Replicate determinations were made using the method reported by Adrian. ${ }^{16}$ The resulting ash was dissolved in $2 \mathrm{ml}$ of concentrated $\mathrm{HNO}_{3}$ and diluted to 100 $\mathrm{ml}$. A $3 \mathrm{ml}$ aliquot of each resultant solution was transferred into a $10 \mathrm{ml}$ calibrated flask and $2 \mathrm{ml}$ of a $5 \%$ La solution were added. The samples were then diluted to volume and analysed by flame AAS against acid-matched standards.

\section{Microwave digestion}

The method used for the closed-vessel microwave digestion of cocoa powder was that described previously. ${ }^{6}$ The method used for the closed-vessel microwave digestion of Horse Kidney was as follows.

A $0.5 \mathrm{~g}$ sample of dried $\left(1 \mathrm{~h}\right.$ at $\left.100^{\circ} \mathrm{C}\right)$ Horse Kidney was accurately weighed into a clean PFA-PTFE digestion vessel and $5 \mathrm{ml}$ of concentrated nitric acid were added. The vessel lid was hand tightened and the carousel containing seven sample vessels and a blank was placed in the microwave oven. One vessel, used for pressure monitoring, was connected to the CEM pressure transducer via a water-filled pressure line. The contents of the vessels were heated according to the programme in Table 3. The vessels were allowed to cool and the resultant solution was transferred into a calibrated flask $(25 \mathrm{ml})$ and diluted to volume. A second dilution was required for the determination of $\mathrm{Ca}$, $\mathrm{Mg}$ and $\mathrm{Zn}$. A $5 \mathrm{ml}$ aliquot of each resultant solution was transferred into a $25 \mathrm{ml}$ calibrated flask and $5 \mathrm{ml}$ of a $5 \% \mathrm{La}$ solution were added. The samples were then diluted to volume and analysed by flame AAS against acid-matched standards.

\section{Slurry preparation}

A sample of cocoa powder ( $1.5 \mathrm{~g})$ was accurately weighed into a clean $10 \mathrm{ml}$ calibrated flask. To the flask were added 7 $\mathrm{ml}$ of a solution of $0.04 \%$ Triton $\mathrm{X}-100$ in $5 \% \mathrm{HNO}_{3}$. The flask was shaken until the solid was homogeneously distributed and the resulting slurry was diluted to volume with $0.04 \%$ Triton $\mathrm{X}-100$ in $5 \% \mathrm{HNO}_{3}$. Horse Kidney and Bituminous Coal slurries consisting of 1.3 and $0.7 \mathrm{~g}$ of the dried sample material, respectively, were prepared in the same way as the cocoa slurries. Attempts were also made to prepare slurries from Pine Needles, Bovine Liver and Oyster Tissue.

\section{On-line digestion}

The on-line digestion protocol developed was as follows The pressure line was filled by pumping concentrated nitric acid through channel $(\mathrm{CH}) 1$ with valves (V) 5,11 and 9 open and $\mathrm{V} 10$ and $\mathrm{V} 7$ closed. When the pressure line was filled, $\mathrm{CH} 1$ was disengaged, V5 and V11 were closed and V 10 was opened. The prepared slurry was transferred into a $5 \mathrm{ml}$ disposable syringe and agitated to ensure suspension of the solid particulates. A $204 \mu \mathrm{l}$ sub-sample of the prepared slurry was introduced into the manifold through the slider valve, V4. A total volume of $1.5 \mathrm{ml}$ of concentrated nitric acid was used to wash the slurry into the digestion column. This volume of acid was introduced into the manifold in two separate stages. Thus, with V1 closed and V2 and V3 open, $\mathrm{CH} 2$ was engaged for the time necessary to deliver 0.8 $\mathrm{ml}$ of acid to the digestion column. This time was set by the flow rate of $\mathrm{CH} 2$ determined prior to the digestion. In order to deliver an equal volume of air into the manifold, V2 was then closed and $\mathrm{V} 1$ opened for the designated length of time. This procedure was repeated such that another $0.8 \mathrm{ml}$ of acid was introduced into the digestion column followed by a slug of air. During this process, the column headspace

Table 3 Digestion programme for the batch microwave digestion of Horse Kidney

$\begin{array}{cccc}\begin{array}{c}\text { Programme } \\ \text { (stage) }\end{array} & \text { Power (\%) } & \begin{array}{c}\text { Pressure/ } \\ \mathrm{lb} \mathrm{in}^{-2}\end{array} & \text { Time/min } \\ 1 & 70 & 50 & 10 \\ 2 & 70 & 100 & 10 \\ 3 & 70 & 150 & 10\end{array}$


Table 4 Digestion programme for the on-line digestion of Horse Kidney and cocoa powder

\begin{tabular}{cccc}
$\begin{array}{c}\text { Programme } \\
\text { (stage) }\end{array}$ & Power (\%) & Pressure/lb in ${ }^{-2}$ & Time/min \\
1 & 50 & 400 & 2 \\
2 & 0 & 300 & 1 \\
3 & 25 & 400 & 3 \\
\hline
\end{tabular}

was allowed to vent through $\mathrm{V} 10$ and $\mathrm{V} 9 . \mathrm{CH} 2$ was then disengaged, V6, V8, and V9 were closed and V11 was opened.

At the conclusion of this process, the slurry and required volume of acid were contained within the column and only the valve leading to the pressure line and transducer was open. A $500 \mathrm{ml}$ water load was placed within the oven cavity. The slurry was digested using the power programme given in Table 4 with subsequent water cooling by the recirculation of ice water pumped through the cooling jacket surrounding the digestion column. Judicious application of microwave energy and continuous monitoring of the system pressure during method development prevented over-pressurization of the digestion manifold. Thus, any potentially dangerous reactions, involving easily oxidized organic material, could be identified and an appropriate programme developed. When the pressure had dropped, V7 and V8 were opened to vent the residual pressure and collect the resultant digest in a $10 \mathrm{ml}$ calibrated flask. V5, $\mathrm{V} 10$ and V11 were opened and the contents of the pressure line were pumped through the transducer, pressure line and digestion column into the collection vessel so as to wash out the system. The collected digest was then diluted to volume and analysed by flame AAS against acid-matched standards.

\section{Residual carbon determination}

The residual carbon content (RCC) of the on-line cocoa powder digests was determined. A $5 \mathrm{ml}$ aliquot of each digest was boiled nearly to dryness, $2 \mathrm{ml}$ of $50 \mu \mathrm{g} \mathrm{ml}^{-1} \mathrm{Sc}$ (prepared from solid $\mathrm{Sc}_{2} \mathrm{O}_{3}$ ) were added as an internal standard and the digest was diluted to $10 \mathrm{ml}$. Carbon emission was measured by ICP-AES at $193.091 \mathrm{~nm}$ to determine the RCC of the digests. The percentage of residual carbon $(\% \mathrm{RC})$ in the digests was determined by expressing as a percentage the ratio of the RCC to the original carbon content of the solid cocoa powder as determined by an elemental analysis performed by an independent microanalytical laboratory.

\section{Evaluation of results}

Statistical treatment of the data was performed using Statview version $1.1 \mathrm{~b} 2$, a commercially available statistical analysis program (Abacus Concepts).

\section{Method Development}

A suitable pressure transducer having a pressure rating of $\geqslant 500 \mathrm{lb} \mathrm{in}^{-2}$ (the maximum operating pressure of the valves) and being chemically inert and capable of direct incorporation into a flowing stream was required (as was also required in the original manifold design $\left.{ }^{6}\right)$. Various materials were investigated for use either as a suitable pressure-sensing diaphragm or as a fill liquid. A PTFE open tubular reactor and a glass column reactor were evaluated for use as digestion vessels within the microwave oven cavity of the flow manifold. The composition of the acid carrier, the position of the digest within the microwave cavity and the use of microwave power programming were studied. A variety of different slurries were prepared and evaluated for manipulation and digestion within the FI manifold. The accuracy of the sub-sampling of the slurry by injection and the homogeneity of the sample material were then assessed by application of the protocol developed to well characterized sample materials.

\section{Results and Discussion}

\section{Pressure Transducer}

Increased pressure within the flow line deforms the PTFE membrane that separates the chemical system from the stainless-steel body of the transducer. The pressure wave is transmitted through a fill liquid, in this instance water, resulting in deflection of the pressure gauge needle. The stainless-steel body provided structural support and a means of transmitting the pressure to the pressure gauge. The displacement volume required to register a pressure of $500 \mathrm{lb}$ in $^{-2}$ on the gauge was determined experimentally and the dimensions of a hemispherical depression into which the PTFE membrane would deform on the application of that pressure were calculated. A PTFE block was fabricated to serve as the face of the pressure transducer.

A number of materials were evaluated for use as a membrane between the flow channels and the transducer body, including CEM rupture discs, 0.001 in stainless-steel shim stock, 0.031 in Viton sheeting, mica sheeting, latex, polyethylene, Mylar and Parafilm. The most satisfactory results were obtained with a PTFE membrane cut by a carbon steel punch $(3.3 \mathrm{~cm}$ diameter) from $24 / 20$ sleeving used for ground glass joints. Prior to use as a membrane, the PTFE must be 'conditioned', or stretched, while in place within the transducer, by the gradual application of increasing pressure. This provides the malleability necessary for constant deformation of the membrane. The major disadvantage of the use of PTFE membranes was their permeability to nitrogen oxides.

A suitable pressure fluid was required to transmit the pressure fluctuations to the pressure gauge. The ideal pressure fluid would have a low viscosity, low compressibility and a low thermal coefficient of expansion. A number of materials were investigated as pressure transmission fluids within the body of the transducer, including silicone oil, automobile transmission fluid and water. Water was found to be the most suitable.

A number of fluids were investigated for transmission of the pressure changes from the digestion column to the pressure transducer, including air, water and nitric acid. The most satisfactory results were obtained using a nitric acid-filled pressure line. Water was found to be unsuitable, as incomplete digestion of the sample material was observed, possibly owing to the dissolution of dinitrogen oxide required to complete the digestion. Its low boiling-point contributed to a rapid pressure increase within the system. Connections between the digestion vessel and the transducer were rated to $1000 \mathrm{lb} \mathrm{in}^{-2}$ and were, in addition, suitably shielded with absorbent material. A catastrophic failure of one of these connections would result in the release of only $1.5 \mathrm{ml}$ of nitric acid. A failure of the membrane would result in a pressure rise within the stainless-steel body of about $50 \mathrm{lb} \mathrm{in}^{-2}$, well within the capabilities of the transducer body and connections. Any runaway over-pressurization could be released via valve V5 (Fig. 2) into an enclosed vessel containing the acid carrier.

\section{Flow Manifold}

Preliminary investigations into the use of microwave heating utilized the manifold described previously ${ }^{6}$ with a 
CEM microwave oven replacing the thermal oven. A single coil of $130 \mathrm{~cm} \times 1.5 \mathrm{~mm}$ i.d. PTFE tubing was looped through the oven cavity. Poly(tetraifluoroethylene) valves at the inlet and outlet ports served to trap the slurry within the oven during the digestion process. Incomplete digestion of the sample material was observed while using this manifold. This was due to the design of the microwave oven in which a substantial length of the flow channels provided is located outside of the oven cavity. Increased pressure resulting from gas evolution during the digestion process forced the slurry into those portions of the flow lines exterior to the oven cavity.

In attempts to improve the quality of digestion, the manifold was modified such that one of the valves was placed within the oven cavity. The second valve and pressure transducer were required to remain outside the cavity owing to the materials from which the transducer was constructed. The injected slurry was then digested in two stages such that the portion of slurry forced out of the cavity during the initial digestion could be swept back into the oven cavity during the collection of the first digest and subsequently decomposed. The two digests were then merged in the collection vessel and diluted to a known volume for flame AAS analysis.

Although the resulting digests appeared to be free from particulate matter, this digestion protocol was laborious. It was also considered desirable to provide some headspace for the expansion of gaseous reaction products to allow for increased sample loading and the decomposition of less reactive materials. It was for these reasons that the closedloop manifold was abandoned and a single-line FI manifold was developed in which digestion of the injected slurry occurred within a glass column mounted vertically within the cavity of the microwave oven. Internal pressure was monitored through the use of the flow-through pressure transducer. A network of PTFE valves was employed for slurry/digest manipulation and controlled depressurization of evolved gases. The digestion column was placed in a number of different locations within the oven cavity, but no evidence of any significant inhomogeneity in the microwave field was observed. The dual-column interface ${ }^{6}$ was not used in this design, as it was initially intended to interface the digestion column directly with the spectrometer. This was later found to be impractical owing to lengthy cool-down periods and digest losses during pressure venting, despite attempts to reduce the internal pressure by cooling the contents of the digestion column.

The proposed flow manifold has a number of advantages over the previously described thermally heated digestion manifold. ${ }^{6}$ The proposed manifold design eliminates the need for merging acid streams as intimate mixing of sample and reagent occur within the digestion column. The column also provides suitable headspace for the expansion of nitrogen oxides, which are believed to play a critical role in the digestion process. Modification of the microwave oven is not required and the digestion column and related valving are mounted on a polystyrene backing such that the assembly is free-standing and can easily be removed from the microwave cavity should batch microwave digestion be desired.

The use of microwave heating and the ability to power programme is an added advantage of the system. When using a thermal oven for sample decomposition, there exists a narrow range of temperatures and residence times for any given material, which may or may not result in complete destruction of the sample matrix. This range is dictated by the pressure limitations of the system and the reactivity of the sample material. At temperatures or residence times beyond this operating range, either the destruction of the sample matrix will be incomplete, owing to insufficient oxidizing power, or the pressurized manifold will become a safety hazard, because without the ability to heat and cool the oven rapidly, the operator has little control over the rate of sample decomposition. These difficulties are easily overcome through the use of a microwave heating source with which the operator completely dictates the energy input, and hence the rate and extent of chemical decomposition.

One consequence of using a sample digestion vessel whose volume is small in comparison with those used in conventional digestion protocols is the reduced mass of sample taken for analysis. As material is introduced as a discrete sub-sample of a prepared slurry, there is an upper limit to the sample mass that can be introduced into the flow manifold. This limit is set by the highest concentration that will produce a stable slurry and by the volume of slurry that can be manipulated within the manifold, and also the requirements of headspace and acid volume for a given sample mass. An acid volume of $2 \mathrm{ml}$ of concentrated nitric acid for a sample mass containing $100 \mathrm{mg}$ of carbon has been found suitable for the decomposition of organic samples. ${ }^{19}$ Likewise, a sample mass containing $1.4-2.8 \mathrm{mg}$ of carbon per millilitre of vessel volume is recommended to obtain optimum decomposition. ${ }^{19}$ Based on these recommended values for acid and vessel volumes, the upper limit for the mass that can be delivered to the digestion column (based on $50 \%$ carbon content and an $8 \mathrm{ml}$ column volume) is $45 \mathrm{mg}$ of sample. The corresponding volume of nitric acid is therefore $0.5 \mathrm{ml}$. Larger volumes of acid are required, however, to ensure transport of the slurry into the digestion column. For cocoa powder and Horse Kidney, the upper slurry concentration limit that can be manipulated within the FI manifold is $15 \% \mathrm{~m} / \mathrm{v}$. The maximum volume of slurry that can be introduced into the digestion column, assuming it can be manipulated within the manifold, is therefore $0.3 \mathrm{ml}$. In the current protocol, $0.2 \mathrm{ml}$ of the slurry is injected into and transported within the manifold. The mass of sample injected is therefore $30 \mathrm{mg}$, thus allowing for some increase in the sample loading. The IAEA recommends that the minimum sample mass of Horse Kidney taken for analysis be $100 \mathrm{mg}$. Clearly this raises concerns over the homogeneity of distribution of minor and trace elements in the sub-samples taken for analysis. This will be discussed later.

On the other hand, if the dilution within the manifold (the $0.2 \mathrm{ml}$ injection volume is diluted to $10 \mathrm{ml}$ ) were less and a detector were used whose detection limits were better than those of the flame atomic absorption instrument used, the mass of sample required could be reduced. This would enable more dilute slurries to be handled, which in turn would increase the range of sample materials that could be handled as stable slurries in the system, but would place additional demands on sample homogeneity. The dilution factor could be reduced by the use of a direct interface with the detector, similar to that reported previously. ${ }^{6}$

\section{Operating Parameters}

The sample mass taken to be slurried was dependent on the concentration of the elements to be determined and the density of the resultant slurry. On-line digestion of some sample materials was not feasible as the mass of material required to provide a trace element content suitable for determination by flame AAS after sample dilution resulted in slurries that could not be manipulated within the confines of an FI manifold. Attempts to slurry Pine Needles, Bovine Liver and Oyster Tissue were unsuccessful, resulting in dense, flocculated suspensions. Similar results have been reported by other researchers ${ }^{15}$ investigating the manipulation of dense slurries in closed systems.

The operating parameters of acid volume and headspace volume were chosen to resemble those determined to be 
Table 5 Determination of minor and trace elements in cocoa powder and Horse Kidney ( $\mu \mathrm{g} \mathrm{g}^{-1}$ in the solid). The \pm terms are $95 \%$ confidence intervals based on the number of replicates given in parentheses

Horse Kidney

\begin{tabular}{lccc}
\cline { 2 - 4 } Element & Microwave & On-line & Certified \\
Iron & $252 \pm 4(n=6)$ & $223 \pm 15(n=5)$ & $265 \pm 15$ \\
Zinc & $204 \pm 4(n=5)$ & $188 \pm 8(n=4)$ & $193 \pm 6$ \\
Cadmium & $\mathrm{NA}^{*}$ & $164 \pm 12(n=4)$ & $189 \pm 4$ \\
Magnesium & $828 \pm 21(n=5)$ & $1053 \pm 145(n=4)$ & $818 \pm 75$ \\
Calcium & $798 \pm 14(n=5)$ & $\mathrm{NA}^{*}$ & $924 \pm 77$ \\
& & Cocoa powder \\
Iron & Microwave & On-line & Hot-plate-ash \\
Magnesium & $189 \pm 10(n=7)$ & $179 \pm 26(n=3)$ & $181 \pm 4(n=3)$ \\
Calcium & $6070 \pm 38(n=7)$ & $6242 \pm 185(n=4)$ & $6076 \pm 33(n=6)$ \\
& $2313 \pm 51(n=7)$ & $1330 \pm 133(n=4)$ & $2148 \pm 18(n=8)$
\end{tabular}

*Not available.

optimum by Wurfels et al.19 for the decomposition of biological samples with acids. The concentrated nitric acid carrier used for the thermal oven experiments was still found to be suitable in the microwave experiments. The use of a sulfuric-nitric acid mixture did not noticeably contribute to the digestion process, and the use of hydrochloricnitric acid mixtures was found to be undesirable owing to rapid pressure increases and the ability of the mixture to digest the stainless-steel body of the pressure transducer.

A number of power programmes were evaluated based on visual observations for the digestion of cocoa slurries. The programme given in Table 4, although not the optimum, was chosen as sufficient for the destruction of all organic matter present in the sample. Occasional shut-down of the magnetron was necessary to prevent over-pressurization of the system, as no pressure feedback is currently available.

\section{Determination of Trace Metals in Cocoa Powder and Horse Kidney}

A summary of the results obtained for the determination of some minor metals in cocoa powder and Horse Kidney is given in Table 5. For the on-line digestion procedure for the Horse Kidney, with the exception of the results for calcium, the $95 \%$ confidence intervals about the measured concentration values overlap the confidence intervals about the certificate values, indicating that there is no statistically significant difference between the values. Although the same situation can be reported for calcium, this arose only because of the very large value for the confidence interval about the measured value. This is discussed below. It can also be seen that there is no significant difference between the results of a batch microwave digestion procedure and the certificate values (apart from the results for calcium). For the cocoa powder, there is no significant difference between the results for the on-line procedure, the batch microwave procedure and the hot-plate-dry ash procedures for iron and magnesium. The results for calcium are again more variable. The precision of the proposed protocol is generally of the order of $10 \%$. This is due to the limitation of flame AAS for the determination of elements in concentrated nitric acid matrices using FI sample introduction, as the presence of the matrix causes a significant shift in the Ringbom plot (relative standard deviation versus concentration) to poorer precisions.

The extent of sample digestion was measured as the percentage of carbon remaining in the sample after decomposition by the proposed method. Determination of residual carbon in five replicate cocoa digests indicated that $12.9 \pm 1.3 \%$ (where the \pm term defines the $95 \%$ confidence interval) of carbon remains in the digest, i.e., approximately $87 \%$ of the original carbon had been lost as $\mathrm{CO}_{2}$.

Further results are given in Tables 6 and 7. If the material exhibited inhomogeneity at the level of the masses introduced for analysis, it might be expected that the withinslurry variance would be larger than the between-slurry variance, if the material were homogeneous at the masses taken to make each $10 \mathrm{ml}$ of slurry. Whether such an effect was observed was evaluated by the use of an analysis of variance (ANOVA) calculation. The situation examined here is different from that normally tested for in a one-way ANOVA, ${ }^{20}$ as in this latter instance the calculations are performed on the basis that the alternative to the null hypothesis is that the between-'batch' variance is larger than the within-batch variance. As it is this version of the calculations that is performed by the Statview program used, the effect of sample inhomogeneity would be to produce an $F$-value of $<1$. The results of the ANOVA calculations are given in Table 8 , from which it can be seen that in only one instance (that of the iron content of Horse Kidney) was this effect observed. For the other elements in these two materials there is an indication that for some determinations the between-slurry variance was significantly larger than the within-slurry variance.

Table 6 Determination of minor and trace elements in cocoa powder by on-line digestion $\left(\mu \mathrm{g} \mathrm{g}^{-1}\right.$ in the solid)

\begin{tabular}{|c|c|c|c|c|}
\hline \multirow{2}{*}{$\begin{array}{c}\text { Replicate } \\
\text { No. }\end{array}$} & \multicolumn{4}{|c|}{ Magnesium } \\
\hline & Slurry 1 & Slurry 2 & Slurry 3 & Slurry 4 \\
\hline $\begin{array}{l}1 \\
2 \\
3\end{array}$ & $\begin{array}{l}6055 \\
5808 \\
5891\end{array}$ & $\begin{array}{l}6049 \\
6003 \\
5911\end{array}$ & $\begin{array}{l}6035 \\
6281 \\
6158\end{array}$ & $\begin{array}{l}6828 \\
6780 \\
7110\end{array}$ \\
\hline \multirow{4}{*}{$\begin{array}{l}\text { Mean } \\
\text { Standard } \\
\text { deviation }\end{array}$} & 5918 & 5988 & 6158 & 6906 \\
\hline & 126 & 70 & 123 & 178 \\
\hline & \multicolumn{4}{|c|}{ Calcium } \\
\hline & Slurry 1 & Slurry 2 & Slurry 3 & Slurry 4 \\
\hline $\begin{array}{l}1 \\
2 \\
3 \\
4 \\
5\end{array}$ & $\begin{array}{r}866 \\
1230 \\
1090 \\
\text { NA* }^{*} \\
\text { NA* }^{*}\end{array}$ & $\begin{array}{l}1577 \\
1239 \\
1267 \\
\text { NA* }^{*} \\
\text { NA* }^{*}\end{array}$ & $\begin{array}{l}1283 \\
1283 \\
1027 \\
\text { NA* }^{*} \\
\text { NA* }^{*}\end{array}$ & $\begin{array}{l}1601 \\
1830 \\
1754 \\
1601 \\
1703\end{array}$ \\
\hline \multirow{2}{*}{$\begin{array}{l}\text { Mean } \\
\text { Standard } \\
\text { deviation }\end{array}$} & 1062 & 1361 & 1198 & 1698 \\
\hline & 183 & 187 & 148 & 99 \\
\hline
\end{tabular}


Table 7 Determination of minor and trace elements in Horse Kidney by on-line digestion ( $\mu \mathrm{g}^{-1}$ in the solid)

Iron

Replicate No.

1
2
3
4

Mean

Standard deviation

1
2
3
4

Mean

Standard deviation

1
2
3
4

Mean

Standard deviation

1
2
3
4

Mean

Standard deviation

\begin{tabular}{ccccc}
\multicolumn{5}{c}{ Iron } \\
\hline Slurry 1 & Slurry 2 & Slurry 3 & Slurry 4 & Slurry 5 \\
215 & 244 & 218 & 212 & 225 \\
215 & 203 & 232 & 226 & 225 \\
215 & 217 & 218 & 226 & 225 \\
257 & 203 & 232 & 226 & 225 \\
225 & 217 & 225 & 223 & 225 \\
21 & 19 & 8 & 7 & 0
\end{tabular}

\begin{tabular}{rrrr}
\hline Slurry 1 & Slurry 2 & Slurry 3 & Slurry 4 \\
188 & 181 & 184 & 187 \\
182 & 194 & 190 & 191 \\
180 & 189 & 192 & 199 \\
175 & 183 & 194 & 193 \\
181 & 187 & 190 & 193 \\
5 & 6 & 4 & 5 \\
& Cadmium &
\end{tabular}

\begin{tabular}{cccc}
\hline Slurry 1 & Slurry 2 & Slurry 3 & Slurry 4 \\
175 & 150 & 146 & 145 \\
181 & 157 & 159 & 155 \\
187 & 163 & 162 & 155 \\
190 & 166 & 166 & 172 \\
183 & 159 & 158 & 157 \\
7 & 7 & 9 & 11 \\
& Magnesium &
\end{tabular}

\begin{tabular}{cccc}
\hline Slurry 1 & Slurry 2 & Slurry 3 & Slurry 4 \\
1269 & 1157 & 1009 & 831 \\
1178 & 1002 & 958 & 816 \\
1192 & 973 & 1163 & 962 \\
NA* $^{*}$ & 988 & NA* $^{*}$ & 1094 \\
1213 & 1030 & 1044 & 926 \\
49 & 85 & 107 & 130
\end{tabular}

*Not available.

Table 8 ANOVA of the results for cocoa powder and Horse Kidney on-line digests

$\begin{array}{lccc}\text { Element } & \text { Sample } & \text { Calculated } F \text {-value* } & \text { Significance } \\ \text { Copper }^{6} & \text { Cocoa powder } & 1.809 & 0.10<p<0.25 \\ \text { Magnesium } & \text { Cocoa powder } & 36.516 & p<0.0001 \\ \text { Calcium } & \text { Cocoa powder } & 13.719 & 0.0001<p<0.005 \\ \text { Iron } & \text { Horse Kidney } & 0.288 & p>0.25 \\ \text { Zinc } & \text { Horse Kidney } & 3.513 & 0.025<p<0.05 \\ \text { Cadmium } & \text { Horse Kidney } & 8.7 & 0.0001<p<0.005 \\ \text { Magnesium } & \text { Horse Kidney } & 4.738 & 0.025<p<0.05 \\ \text { Calcium } & \text { Horse Kidney } & 3.285 & 0.10<p<0.25\end{array}$

*Calculated $F$-value.

†Probability of the calculated $F$-value occurring by chance if the null hypothesis is valid.

Difficulties arose during the determination of iron and calcium in the digested samples. The determination of iron in cocoa powder was carried out by ICP-AES rather than flame AAS owing to the presence of an interferent in the digest matrix. This interference can be overcome in the flame determination through the use of the method of standard additions or, alternatively, through the use of dinitrogen oxide-acetylene flame. However, for the samples used in this study the concentration of iron in the resulting digest was too low to be determined reliably because of the lower sensitivity of the dinitrogen oxide-acetylene flame in comparison with that of an air-acetylene flame.

The determination of calcium in both cocoa powder and
Horse Kidney was found to be problematic for samples digested using the proposed manifold. The recoveries were generally found to be low and variable. Blank values were observed to be erratic and digestion of an aqueous calcium standard in the manifold yielded inconsistent results. It is believed that this behaviour is the result of interaction between the calcium in the sample and the borosilicate glass column occurring at elevated temperature and pressure, as no losses were observed when a calcium standard was exposed to the column under ambient conditions. It is therefore not feasible to employ the manifold for the determination of calcium in solid samples.

Another limitation of the proposed system was encoun- 
tered during the digestion of SRM 1632b Bituminous Coal. An attempt was made to leach out a number of elements of interest from the sample material without the use of hydrofluoric acid in order to prevent degradation of the glass digestion column. The sample was prepared and digested in the same manner as the Horse Kidney and cocoa powder. As expected, the resultant digest contained residual silicatebased particulates, requiring that the digests be filtered prior to analysis. Analysis of the resultant digests yielded imprecise results with large confidence intervals. It is concluded that the acid leach was unsuccessful, with varying concentrations of the trace metals remaining bound to the undigested silicate material. This limitation of the system might possibly be overcome through the use of an alternative material for construction of the digestion column.

\section{Practical Considerations}

The manifold developed requires a certain degree of routine maintenance in order to function efficiently. The Viton $\mathrm{O}-$ rings that seal the glass column end-fittings need to be replaced approximately once every 2 weeks of constant use. The PTFE valves also have a finite lifetime and require replacement approximately every $2-3$ months to prevent leakage and hence loss of digest. Problems have arisen within the manifold owing to the materials from which the slider valve is constructed. The Tefzel body and flow lines within the valve are not very resistant to oxidation by concentrated nitric acid and require frequent changing. As this becomes costly, PTFE equivalent counterparts to the Tefzel components have been constructed and are currently in use. The final consideration is the design of the microwave oven. The placement of the flow lines in the CEM oven currently employed is not very suitable for the proposed manifold design. The geometry of the flow lines means that the slurry is transported a substantial distance external to the microwave cavity prior to entering the digestion column. This requires the use of large volumes of acid to wash the slurry adequately from the manifold tubing. This property of the oven design also creates a substantial back-pressure which must be overcome in order to manipulate the slurry successfully within the manifold. The modification of a domestic microwave oven to alleviate these shortcomings is currently under consideration.

\section{Conclusion}

The ability of the system to operate at pressures in excess of $400 \mathrm{lb}$ in $^{-2}$ allows for the rapid decomposition of organic matrices with subsequent trace metal determinations without the need for any further sample pre-treatment. No difficulties have yet been encountered with regard to sample homogeneity, although the sample amounts used in the system are lower than those recommended. Development of a suitable interface and the use of alternative detection schemes, which provide increased sensitivity over that of the flame AAS procedure, will allow the use of more dilute slurries in order to facilitate slurry manipulation within the manifold. The move to alternative detectors may also provide for a wider range of applications to sample materials for which the preparation and manipulation of a concentrated slurry are not feasible.

The authors gratefully acknowledge Richard Zink for assistance in the design and fabrication of the pressure transducer and Dr. Susan Darke for many helpful discussions. Financial support from the University of Massachusetts, Amherst, USA in the form of a faculty research grant is also gratefully acknowledged.

\section{References}

1 Sample Introduction in Atomic Spectroscopy, ed. Sneddon, J., Elsevier, Amsterdam, 1990.

2 Greenfield, S., Hieftje, G. M., Omenetto, N., Scheeline, A., and Slavin, W., Anal. Chim. Acta., 1986, 180, 69.

3 Valcarcel, M., and Luque de Castro, M. D., Automatic Methods of Chemical Analysis, Elsevier, Amsterdam, 1988.

4 Rủžička, J., and Hansen, E. H., Flow Injection Analysis, Wiley, New York, 2nd edn., 1988.

5 Tyson, J. F., Spectrochim. Acta Rev., 1991, 14, 169.

6 Gluodenis, T. J., Jr., and Tyson, J. F., J. Anal. at. Spectrom., 1992, 7, 301.

7 Hinkamp, S., and Schwedt, G., Anal. Chim. Acta, 1990, 236, 345.

8 Balconi, M. L., Borgarello, M., Ferraroli, R., and Realini, F., Anal. Chim. Acta, 1992, 261, 295.

9 Tsalev, D. L., Sperling, M., and Welz, B., in Proceedings of the Sixth Colloquium Atomspekrometrische Spurenanalytik, Konstanz, 8-12 April, 1991, ed. Welz, B., Perkin-Elmer, Überlingen, 1991, pp. 349-366.

10 Welz, B., Tsalev, D. L., and Sperling, M., Anal. Chim. Acta, 1992, 261, 91.

11 Burguera, M., Burguera, J. L., and Alarcon, O. M., Anal. Chim. Acta, 1988, 214, 421.

12 de la Guardia, M., Salvador, A., Burguera, J. L., and Burguera, M., J. Flow Injection Anal., 1988, 5, 121.

13 Carbonell, V., de la Guerdia, M., Salvador, A., Buguera, J. L., and Burguera, M., Anal. Chim. Acta, 1990, 238, 417.

14 Karanassios, V., Li, F. H., Liu, B., and Salin, E., J. Anal. At. Spectrom., 1991, 6, 457.

15 Haswell, S. J., and Barclay, D., Analyst, 1992, 117, 117.

16 Adrian, W. J., Analyst, 1973, 98, 213.

17 Issac, R. A., and Johnson, W. C., J. Assoc. Off. Anal. Chem., $1975,58,436$.

18 Iverson, J., J. Assoc. Off. Anal. Chem., 1972, 55, 1027.

19 Wurfels, M., Jackwerth, E., and Stoeppler, M., Anal. Chim. Acta, 1989, 226, 1.

20 Miller, J. C., and Miller, J. N., Statistics for Analytical Chemistry, Ellis Horwood, Chichester, 1984.

NotE-Ref. 6 is to Part 1 of this series. 